\title{
Settlement patterns and the eastern boundary of the Northern Cities Shift - ERRATUM
}

\author{
Aaron J. Dinkin
}

doi:10.1017/jlg.2013.2, Published by Cambridge University Press, 29 August 2013.

An error was made in the first paragraph of Section 4.2 (page 14) of the above publication. The sentence which reads:

"In the six communities on the left side of Figure 12-Utica, Gloversville, Sidney, Watertown, Glens Falls, and Ogdensburg-all speakers in the data have EQ1 indices greater than or equal to 88."

Should read:

"In the six communities on the left side of Figure 12-Utica, Gloversville, Sidney, Watertown, Glens Falls, and Ogdensburg-all speakers in the data have EQ1 indices greater than or equal to -88."

\section{Reference}

Aaron, J. Dinkin. 2013. Settlement patterns and the eastern boundary of the Northern Cities Shift. Journal of Linguistic Geography 1: 4-30, doi:10.1017/jlg.2013.2. 